\title{
Estilos de aprendizaje predominantes relacionado al uso de las MOOC a través de la regresión logística
}

\section{Predominant learning styles related to the use of MOOCS through the logistic regression}

\author{
Angela Torres Rivera ${ }^{1}$ \\ Doris Elida Fuster-Guillén² \\ Yudith Ivonne Alata Cusy ${ }^{3}$ \\ Sandy Dorian Isla Alcoser ${ }^{4}$
}

Recibido: 20 de diciembre de 2019. Aceptado: 14 de enero de 2020

\section{RESUMEN}

A nivel pedagógico, la identificación de los estilos de aprendizaje ha sido una estrategia de éxito en la enseñanza. En estos tiempos nos enfrentamos a nuevos espacios de aprendizaje, como son los MOOC. El estudio tiene como objetivo identificar el estilo de aprendizaje predominante en el uso de los $\mathrm{MOOC}$ en los alumnos de maestría de la Universidad Nacional Mayor de San Marcos (UNMSM), Lima, 2019-I. La investigación es de enfoque cuantitativo, método hipotético deductivo, diseño no experimental, corte transversal y nivel explicativo, y se basa en una muestra de 111 alumnos.

Palabras claves: MOOC; estilos de aprendizaje; educación; Maestría.

\begin{abstract}
At pedagogical context, identifying learning styles has been a successful teaching strategy. Nowadays, we have new learning spaces such as MOOC. The research aims to identify the predominant learning style in use of MOOC for the master degree's students at National Major University of San Marcos (UNMSM), Lima, 2019-I. The research is quantitative approach, hypothetical deductive method, non-experimental design, cross section and explanatory level, and is based on a sample of 111 students.
\end{abstract}

Keywords: MOOC; learning styles; education; Master degree.

1 Universidad Nacional Mayor de San Marcos. Correo electrónico: angetorres1401@gmail.com, ORCID: https://orcid.org/0000-0001-7529-9586

2 Universidad Nacional Mayor de San Marcos. Correo electrónico: dfusterg@unmsm.edu.pe, ORCID: https://orcid.org/0000-0002-7889-2243

3 Universidad Nacional Mayor de San Marcos. Correo electrónico: yalatac@unmsm.edu.pe, ORCID: https://orcid.org/0000-0001-9123-976X

4 Universidad Nacional Mayor de San Marcos. Correo electrónico: sislaa@unmsm.edu.pe, ORCID: https://orcid.org/0000-0003-1330-3716

(c) 2020 Revista Multi-Ensayos. 


\section{INTRODUCCIÓN}

En el tiempo se ha revisado la importancia de la identificación de los estilos de aprendizaje como estrategia de éxito para la transmisión de conocimiento (Ceballos y Arribas, 2003; González, Patarroyo y Carreño, 2017; Rodríguez, 2018), y ha sido puesto en práctica a través de evaluaciones en aulas tradicionales (García, Barrantes, Ureña y Mora, 2015) con resultados significativos. Sin embargo hoy, la comunidad académica pone énfasis en la expansión de conocimiento a través de nuevos medios como las MOOC (Ruíz, 2015), pero a su vez cuestionan los criterios para el éxito del mismo. Desde su aparición, se han observado las altas tasas de abandono (Poy y Gonzales-Aguilar, 2014) cuestionando que estos se deberían a características propias del contenido del curso o del medio (Sandoval, Morales, Hernández y AmadoSalvatierra, 2018), o características propias del alumno (Sánchez y Prendes, 2014) como la autoconfianza, el autocontrol, la motivación y las habilidades comunicativas. Sin lugar a dudas, es necesario ahondar en las posibles causas de la baja utilización de los MOOC, de lo contrario los esfuerzos futuros seguirán siendo mermados por la alta de deserción que se viene presentando, las cuales indican llegar al 90\% (Gregori, Martínez y Moyano, 2018).

Se realiza esta investigación para identificar el estilo de aprendizaje que predomina en el uso de los MOOC, con el propósito de que puedan ser consideradas tanto en el desarrollo pedagógico de los cursos MOOC, como en la identificación del público que pueda obtener mayor provecho de este moderno medio de aprendizaje. Los estilos de aprendizaje han sido descritos por distintos, en esta investigación se decidió el planteamiento de David Kolb debido a que está orientado a personas de nivel adulto (Kolb, 1983) y presenta una relación entre aprendizaje y práctica (Herrera L. y De la Hoz A., 2014).

Esta teoría define el aprendizaje de manera experiencial, como un proceso mediante el cual el conocimiento es creado por la transformación de la experiencia concreta del sujeto. Esta visión del aprendizaje estuvo fuertemente influenciada por John Dewey (Ruiz, 2013) quien indicaba que la educación es una constante reorganización y reconstrucción de la experiencia. En base a esta influencia, la teoría de Kolb enfoca el aprendizaje fruto de la herencia, las experiencias anteriores, y las exigencias actuales del ambiente en el que se mueve (Martín, 2003).

En el enfoque de Kolb (Kolb, 1983) la capacidad de adaptación está relacionada a una competencia con la cual la persona puede trabajar o enfrentar los desafíos del aprendizaje de acuerdo al estilo que le predomina. Estas destrezas o competencias se relacionan con características de cada estilo de aprendizaje. Así tenemos que en el estilo acomodador, predominan las competencias de "acción"; en el estilo divergente, las competencias de "valorar"; en el estilo asimilador, las competencias de "pensar"; y en el estilo convergente, las competencias de "decisión". El diagnóstico es mediante el cuestionario Learning Style Inventory (LSI), el cual es un instrumento que facilita cuatro puntuaciones y es posible identificar cada capacidad. Según Kolb (citado en Gutarra y Palomino, 2015) son la capacidad de Experiencia Concreta (EC) donde en el aprender se identifica con los sentimientos, enfatizando la relación personal con la gente en situaciones cotidianas; la segunda capacidad es de Observación Reflexiva (OR), donde el aprender se identifica con la comprensión de ideas y situaciones desde distintos puntos de vista; la tercera capacidad 
es de Conceptualización Abstracta (CA), donde el aprender se identifica con el razonamiento, e implica el uso de la lógica y de las ideas, más que los sentimientos, para comprender los problemas o las situaciones; y la cuarta capacidad es de Experimentación Activa (EA), donde el aprender se identifica con la acción, el aprendizaje se experimenta con el hecho de influir o cambiar situaciones, en oposición a la mera observación. De estas capacidades se desprenden los cuatro estilos de aprendizaje (Kolb D., Boyatzis R. y Mainemelis C., 1999), estas son; el estilo Divergente, donde predominan las capacidades EC y OR; el estilo Asimilador, donde predominan las capacidades CA y OR; el estilo Convergente, donde predominan las capacidades CA y EA; y el estilo Acomodador, donde predominan las capacidades EC y EA.

Los MOOC, conocido por sus siglas como Massive Open Online Courses, aparecieron hace poco más de una década (Cabero J. y Llorente M.,2017), el primer registro que se tiene es en el 2008 George Siemens de la Universidad de Athabasca - Canadá y Stephen Downes del Consejo Nacional de Investigación de Canadá, presentaron el curso "Conectivismo y Conocimiento Conectivo" en modalidad presencial y online donde ofrecían videos, blogs de discusión e intercambio en redes sociales. Luego, Dave Comier de la Universidad de la Isla del Príncipe Eduardo- Canadá llamó a esta experiencia MOOC (Adesope y Rud, 2018). Años más tarde, en el 2013, se lanzaría la plataforma Miriadax que consiguió que se inscribieran en su primera convocatoria 188,802 alumnos; y la extensión de Coursera, plataforma MOOC lanzada años antes, llegó a contar con 22 millones de inscripciones con alumnos de 190 países (Fundación Telefónica, 2015). Los MOOC están siendo estudiados por la comunidad científica como un campo emergente de divulgación de conocimiento que se encuentra en expansión (Cabero, 2015; Gallego, Gámiz y Gutiérrez, 2015).

La definición de MOOC (Castaño y Cabero, 2013) es de un recurso educativo que tiene cierta semejanza con una clase o aula, cuenta con fechas de inicio y finalización, cuenta con mecanismos de evaluación, es a través de internet, no tiene criterios de admisión y permite la participación interactiva a gran escala de cientos de estudiantes. Estas plataformas cuentan con una oferta muy variada, los campos donde se ofrecen cursos van desde las ciencias puras, ciencias sociales y hasta infinidad de cursos del campo técnico (Bulfin, Pangrazio y Selwyn, 2014).

Este nuevo medio permite una educación online en tiempos donde la conectividad digital se extiende rápidamente, brindando acceso a conocimiento que de otro modo sería demasiado costoso o inalcanzable para la mayor parte de la población, esta una de las principales ventajas de este nuevo medio y es señalada como una opción válida para la democratización de la educación (González S., Del Pozo F., Paredes W. y Del Pozo H., 2018). El OCDE (Centro de Desarrollo, 2015) señala que la esperanza es que los MOOC puedan llevar la mejor educación a los rincones más remotos del mundo, a un costo cero o muy bajo para los estudiantes. Esto es especialmente importante para países con restricciones financieras donde el acceso a la educación implica matrículas muy elevadas, y como resultado es cada vez más inasequible y discriminador el acceso a la educación superior. 


\section{DESARROLLO}

El estudio se desarrolló bajo el enfoque cuantitativo (Hernández, Fernández y Baptista, 2010), se utilizó la recolección de datos, medición numérica y el análisis estadístico. El método es hipotético deductivo (Cegarra, 2012), se planteó hipótesis acerca de las posibles soluciones al problema. El diseño es no experimental y de corte transversal (Hernández et al., 2010), debido a que se realiza sin influir o manipular en forma intencionada o deliberada las variables de estudio, y el levantamiento de información es realizado en un lapso especifico de tiempo.

El nivel es explicativo (Sánchez y Reyes, 2015), ya que se hizo necesaria la formulación de un sistema de hipótesis que permitan dar una explicación tentativa. Se contó con una población constituida por 155 estudiantes, los cuales son alumnos de maestría en educación de la Universidad Nacional Mayor de San Marcos (UNMSM) que cursaron estudios durante el período 2019-I. Según muestreo probabilístico aleatorio simple (Sánchez et al, 2015), la muestra fue calculada en 111 encuestados. Y se utilizó como técnica la encuesta (Hurtado, 2000) debido a que la investigación plantea problemas que puede analizarse a través del conocimiento de la experiencia de otras personas. En este caso, era lo más pertinente para poder conocer el estilo de aprendizaje y el nivel de uso de los MOOC por parte de los alumnos.

En la recolección de información para estilos de aprendizaje se consideró la utilización del cuestionario de identificación o inventario de estilos de aprendizaje LSI. Este está constituido por 4 columnas que están asociadas a cada uno de los cuatro indicadores (capacidades) que conforman el aprendizaje experiencial, cada una de ellas conformadas a su vez por 12 ítems que simulan o representan diversas situaciones de aprendizaje vinculadas a cuatro capacidades: Experiencia Concreta (EC), Observación Reflexiva (OR), Conceptualización Abstracta (CA), Experimentación Activa (EA). La validez y confiabilidad del instrumento ha sido sustentado por Kolb (Delgado, 2004), donde realizó una prueba sobre 287 individuos para analizar la validez de las dimensiones relacionando los puntajes asignados a las palabras con su correspondiente fase de aprendizaje, alcanzando correlaciones de Pearson que van desde $r=0.50$ a $r=0.60$, llegando a la conclusión que el test tenía validez.

Asimismo, se evaluó la confiabilidad del instrumento, utilizando el método de la división por mitades (Split-Half), la cual fue computada en forma independiente para cada una de las 4 dimensiones de su instrumento; los resultados arrojados indicaron que las correlaciones oscilaban entre 0.55 y 0.82 en los cinco grupos que se estudiaron. Así mismo, en cuatro de los cinco grupos estudió la estabilidad en el tiempo bajo la modalidad test-retest, encontrando que las relaciones iban desde 0.40 hasta 0.70 ; con estos resultados pudo concluir que su instrumento sí era confiable. Adicionalmente el instrumento ha demostrado validez y confiabilidad en otros estudios de ámbito académico (Cano, 1997; González, 1983). Para la recolección de información de la variable uso de los MOOC se elaboró un cuestionario que consta de 8 ítems, donde cada ítem presenta los siguientes rangos: bajo uso, medio uso, alto uso, realizado a través de la técnica de baremación. 
El instrumento pasó por la validez de contenido (Ding y Hershberger, 2002), teniendo como resultado válido. Para la evaluación de la confiabilidad se realizó una prueba piloto, que permitió la validación del constructo (Lawshe, 1975) y la confiabilidad se analizó con el análisis estadístico del alfa de Cronbach (Cronbach, 1951) cuyo resultado fue 0.817 , con lo que se concluye que el instrumento presenta alta confiabilidad y coherencia interna y en el análisis de resultados de la investigación se utilizó un estadígrafo no paramétrico denominado regresión logística (Maurandi-López, Del Río, González-Vidal, Ferre y Hernández, 2019), debido a los resultados no normales arrojados por la prueba de Kolmogorov Smirnov (Heuser, Rioul y Guilley, 2014), considerando el tamaño de la muestra de 111 encuestas como grande (Burdenski, 2000).

\section{RESULTADOS}

A continuación, se procede a graficar los resultados obtenidos a partir del análisis de frecuencias en las respuestas obtenidas de la muestra de 111 estudiantes:

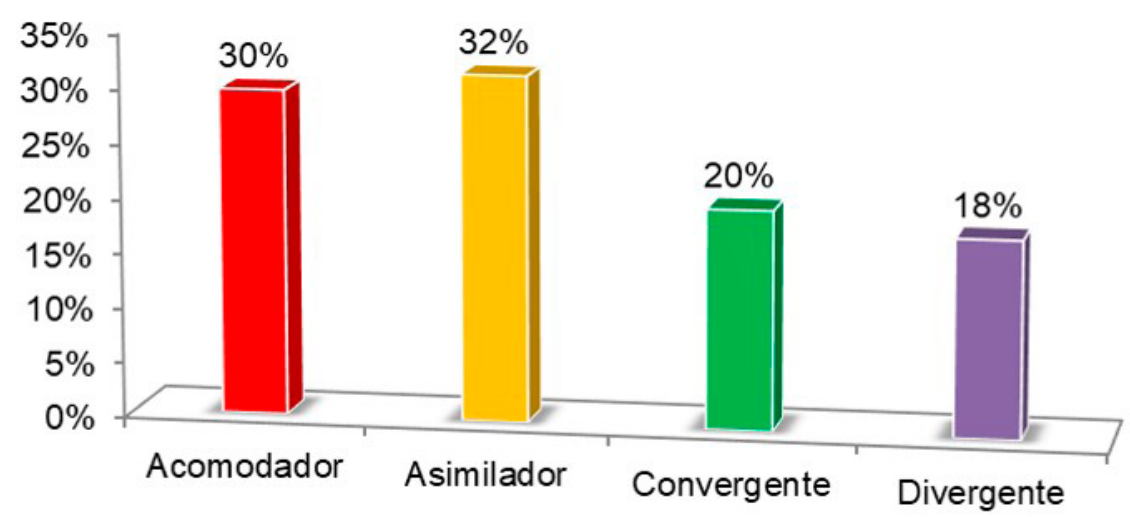

Figura 1. Estilo de aprendizaje predominante en los estudiantes de la maestría en educación de la UNMSM, 2019-I

De la tabla y figura 1, se observa que el $32 \%$ de la muestra presentan el estilo de aprendizaje asimilador como predominante, mientras que el $18 \%$ de los mismos presentan el estilo de aprendizaje divergente como predominante. Este resultado coincide con la investigación de Escurra (1991) quien encontró que el estilo predominante en los alumnos de la UNMSM es el estilo asimilador. La coincidencia de resultados nos demuestra que este estilo predominante está arraigado a la población en análisis.

A través del análisis multivariado, regresión logística, se procede a identificar los estilos predominantes en el uso de las MOOC. 
Tabla 1 Coeficientes de la regresión logística de los estilos de aprendizaje relacionados al uso de las MOOC en los estudiantes de la maestría en educación de la UNMSM, 2019-I

\begin{tabular}{|c|c|c|c|c|c|c|}
\hline \multirow{2}{*}{ Dimensión } & \multirow{2}{*}{ B } & \multirow{2}{*}{ Error estándar } & \multirow{2}{*}{ Sig. } & \multirow{2}{*}{$\operatorname{Exp}(B)$} & \multicolumn{2}{|c|}{ 95\% C.I. para EXP (B) } \\
\hline & & & & & Inferior & Superior \\
\hline Acomodador & 3.466 & 1.016 & 0.001 & 32.000 & 4.373 & 234.180 \\
\hline Asimilador & 0.405 & 0.345 & 0.240 & 1.500 & 0.763 & 2.950 \\
\hline Convergente & -2.351 & 0.740 & 0.001 & 0.095 & 0.022 & 0.406 \\
\hline Divergente & -0.201 & 0.449 & 0.655 & 0.818 & 0.339 & 1.974 \\
\hline
\end{tabular}

El valor de significación observada (Sig.) es mayor al valor de significación teórica $\alpha=0.05$ en dos estilos, se determina que si existen diferencias en los estilos de aprendizaje relacionados al uso de las MOOC. Además se observa que el estilo de aprendizaje acomodador $(B=3,466)$ presenta mayor coeficiente y por ende aporta más a la relación del uso de las $\mathrm{MOOC}$ en los estudiantes de la maestría en Educación de la UNMSM 2019-I. Así mismo, este estilo presenta un odds ratio $(\operatorname{Exp}(B))=32.000$, significando que es de riesgo; es decir, que si el estudiante no refuerza las características o las capacidades de experiencia concreta y experimentación activa; entonces, su nivel de uso de las MOOC podría ser bajo.

En el análisis de los indicadores predominantes por estilo de aprendizaje relacionado al uso de los MOOC en los estudiantes de maestría en educación de la UNMSM, 2019-1, tenemos los siguientes resultados, que aunque no han mostrado una significancia estadística procedemos a detallarlos:

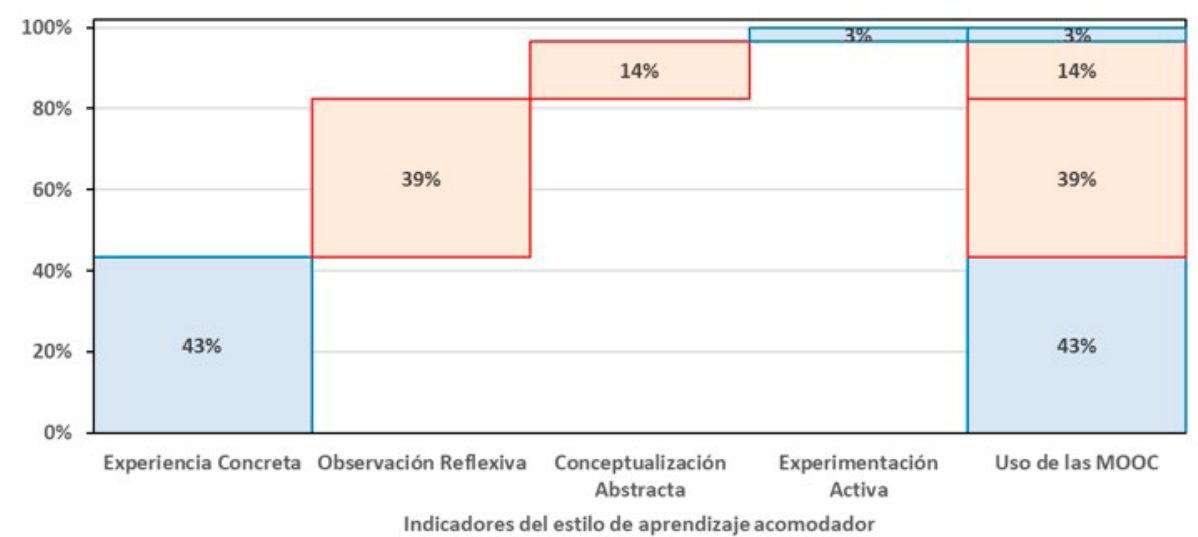

Figura 2. Pesos de los indicadores del estilo de aprendizaje acomodador relacionado al uso de las MOOC en los estudiantes de la maestría en educación de una universidad estatal de Lima, 2019-I

En el estilo de aprendizaje acomodador relacionado al uso de las $\mathrm{MOOC}$, el indicador de mayor peso es la experiencia concreta. $Y$ el indicador de experiencia activa solo ejerce un peso del 3\%, ello nos permite pensar que es el factor necesario a reforzar para poder reducir el riesgo de bajo uso de los MOOC. 


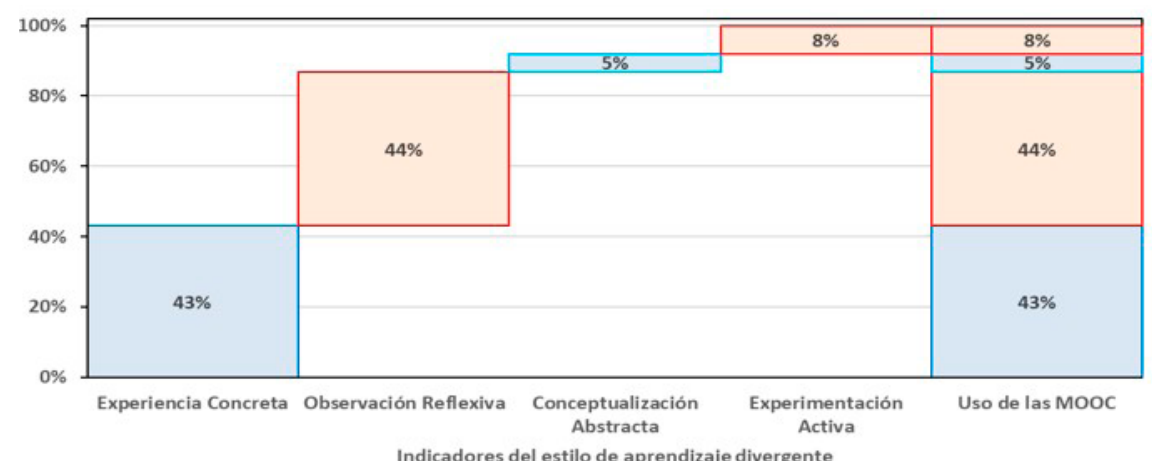

Figura 3. Pesos de los indicadores del estilo de aprendizaje divergente relacionado al uso de las MOOC en los estudiantes de la maestría en educación de la UNMSM, 2019-I

En el estilo de aprendizaje divergente relacionado al uso de las $\mathrm{MOOC}$, el indicador de mayor peso es la observación reflexiva, aunque la diferencia con el peso que ejerce la experiencia concreta es de solo 1\%, por lo que podemos seguir afirmando que la experiencia concreta aporta en buena medida al uso de los MOOC.

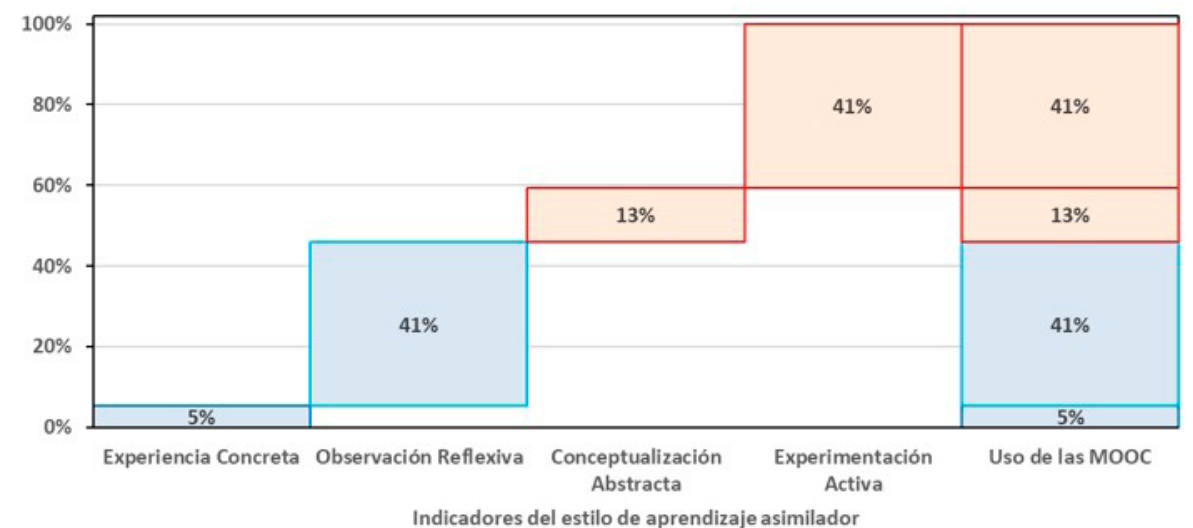

Figura 4. Pesos de los indicadores del estilo de aprendizaje asimilador relacionado al uso de las MOOC en los estudiantes de la maestría en educación de la UNMSM, 2019-I

En el estilo de aprendizaje asimilador relacionado al uso de las MOOC, el indicador de mayor peso es la observación reflexiva, pero a su vez debemos recordar que el aprendizaje asimilador no ejerce mayor peso en relación al uso de las MOOC.

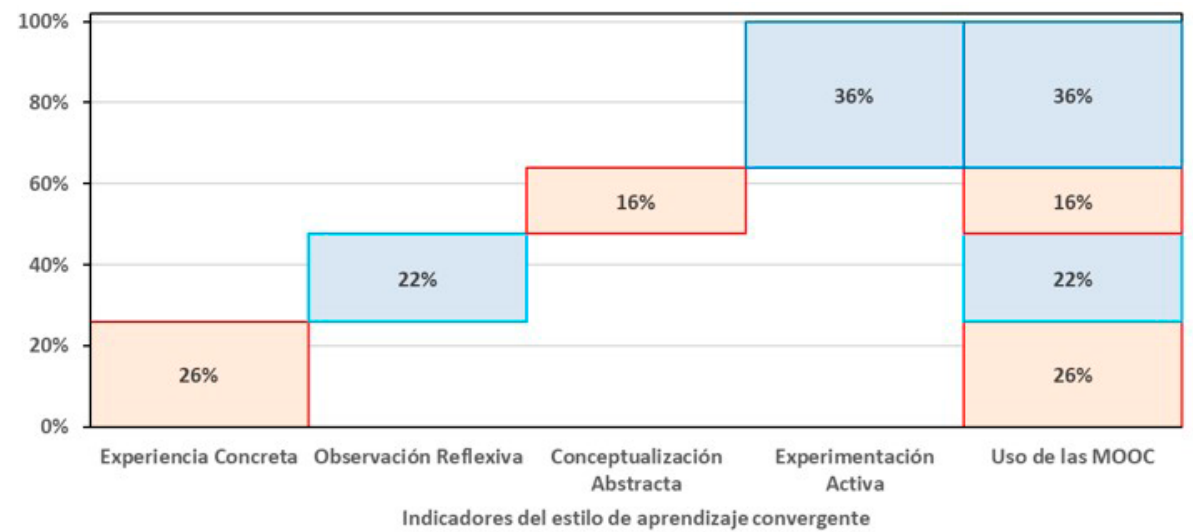

Figura 5. Pesos de los indicadores del estilo de aprendizaje convergente relacionado al uso de las MOOC 
en los estudiantes de la maestría en educación de la UNMSM, 2019-I

En el estilo de aprendizaje convergente relacionado al uso de las $\mathrm{MOOC}$, el indicador de mayor peso es la experimentación activa, que según el análisis previo, es uno de los dos indicadores que se deben reforzar para reducir el riesgo de un bajo uso de los MOOC.

\section{CONCLUSIÓN}

En los hallazgos de la investigación se muestra que el estilo de mayor frecuencia en la población es el Asimilador, sin embargo presenta un bajo peso como estilo de aprendizaje relacionado al uso de los MOOC, esto significa que en la mayoría de la población no presentará un uso significativo de los MOOC.

Esto puede deberse a las características propias de este estilo que son definidas como personas que les preocupa más los conceptos teóricos y disfrutan del análisis y reflexión. Por lo que deducimos que, sobre ellos pueda predominar el estilo clásico de aprendizaje llevado a cabo en aulas tradicionales, donde pueden hacer uso de habilidades que manejan con gusto como la investigación y conceptualización de conceptos.

En concordancia a la investigación realizada por Escurra (1991), donde encontró que en los alumnos de UNMSM predomina el estilo de aprendizaje Asimilador. Entonces podemos confirmar que los datos de la investigación tienen concordancia, y las características de los alumnos se ha mantenido en el tiempo.

Los alumnos con estas características se adaptan mejor a la metodología que ofrece la universidad tradicional y por ello su presencia mayoritaria, a ellos el uso de las MOOC es indiferente. Como docentes, el conocer esta realidad nos permite plantearnos qué características de los MOOC se debería ofrecer para que este tipo de alumnos puedan seguir adquiriendo conocimiento desde la ubicuidad, estamos hablando de la personalización de la masificación que plantea Torres, Infante y Valdiviezo (2014). Esto es importante porque es claro que la permanencia de los alumnos en las aulas no será eterno y la capacitación constante o extensión universitaria es necesaria para que ellos no pierdan vigencia profesional.

En la investigación realizado por Álvarez y Domínguez (2001) encontró que los alumnos de maestría en Administración Educativa de una universidad particular de Lima muestran preferencia por el estilo Asimilador y Acomodador, en ese orden. Esto coincide con los resultados de esta investigación.

Al ser estilos opuestos, tener preferencia por la teoría en el primero y preferencias por la práctica en el segundo, debemos hacer notar que la población en estudio son estudiantes de maestría y no todos cuentan con la carrera profesional de educadores, sino que pueden ser filósofos, ingenieros, artistas, etc., que desean incorporar o adquirir conocimientos de pedagogía o gestión educativa como parte de su desarrollo profesional. Gordillo, Antelo, García y Sayago (2013) en su investigación realizada sobre los estilos de aprendizaje y su implicación en el uso de las TIC y aprendizaje colaborativo, concluye que el indicador predominante para un uso favorable de las TICs es la observación reflexiva, lo cual puede ser sustentado con las herramientas TIC que son parte de su evaluación como el correo electrónico, la biblioteca virtual y las publicaciones web, que tienden a ser espacios donde la interacción fluida no es 
una característica.

Hacemos notar que las nuevas tendencias de MOOC cuentan con un enfoque conectivista (Borrás 2019) ofreciendo un abanico mayor en cuanto a herramientas, por lo cual no es raro que puedan contar con el uso de redes sociales, transmisión de clases a través de video o transmisión en línea con intercambio de información en vivo, esto hace que sea una experiencia más práctica. Por ello nos es consistente, que el estilo predominante en esta investigación en relación al uso de los MOOC sea el estilo acomodador.

En lo que respecta a las limitaciones del estudio, se encuentra que el método de identificación de las variables ha sido a través de cuestionarios, que a pesar de estar validados, dependen de la disposición del estudiante, lo que puede brindar un sesgo a la información. Ello sugiere, que a futuro, se pueda contrastar los resultados obtenidos en la presente investigación con otros instrumentos que podrían ser de características cualitativas como entrevistas, diarios, u otros instrumentos que permitan complementar la información obtenida.

Con respecto a la prospectiva de investigación, el estudio realizado indica la necesidad de seguirinvestigando e indagando sobre este tema, el presente estudio ha identificado los indicadores predominantes en los estilos de aprendizaje relacionado al uso de los $\mathrm{MOOC}$ bajo un estadígrafo no paramétrico, debido a que la información no muestra normalidad. Se recomienda a futuras investigaciones poder ampliar el tamaño de la muestra censal, o llevar el estudio a una población más amplia, donde también se pueda considerar preparar un modelo predictivo con respecto al uso de los MOOC.

Por lo mencionado anteriormente, la importancia de ahondar en el uso de los MOOC desde las características intrínsecas del alumno resulta necesario, pues es un medio que se debe aprovechar para adquirir conocimiento adicional o extensivo a las aulas tradicionales. Esto en el largo plazo será una necesidad imperante en el crecimiento profesional que cada individuo tiene obligación de desarrollar.

\section{REFERENCAIS}

Adesope O. y Rud A.(2018). Contemporary Technologies in Education: Maximizing Student Engagement, Motivation, and Learning. Springer. doi: https://doi.org/10.1007/978-3-319-89680-9

Álvarez D. y Domínguez J. (2001). Estilos de aprendizaje en estudiantes de posgrado de una universidad particular. Persona, (004), 179-200.

Borrás O. (2019). Empowering MOOC Participants: Dynamic Content Adaptation Through External Tools. In European MOOCs Stakeholders Summit (121-130). Springer, Cham. doi: https://doi. org/10.1007/978-3-030-19875-6_14

Bulfin S., Pangrazio L. y Selwyn N. (2014). Making 'MOOCs': The construction of a new digital higher education within news media discourse. The International Review of Research in Open and Distributed Learning, 15(5). doi: https://doi.org/10.19173/irrodl.v15i5.1856

Burdenski Jr, T. K. (2000). Evaluating Univariate, Bivariate, and Multivariate Normality Using Graphical Procedures. Recuperado de https://eric.ed.gov/?id=ED440989 
Cabero J. (2015). Visiones educativas sobre los MOOC. Revista Iberoamericana de Educación a Distancia, 18 (2), 39-60. doi: http://dx.doi.org/10.5944/ried.18.2.13718

Cabero J. y Llorente M. (2017). Los MOOC: encontrando su camino. @tic. revista d'innovació educativa, 18, 24-30. doi: https://doi.org/10.7203/attic.18.9928

Cano, F. (1997). Fiabilidad y validez de constructo del L.S.Q. (Cuestionario de estilos de aprendizaje). Anales de la Revista de Psicología General y Aplicada - IberPsicología, 2.1.4

Castaño C. y Cabero J. (2013). Enseñar y aprender en entornos m-learning. Madrid: Síntesis.

Ceballos I. y Arribas C. (2003). El diagnóstico de los estilos de aprendizaje: un instrumento valioso para apoyar la formación de los estudiantes de enfermería. Revista Enfermería Global, 2(3).

Cegarra, S. (2012). Los métodos de investigación. (1ed.). México: Pearson Educación de México S.A.

Centro de Desarrollo (2015) La educación a distancia en la educación superior en América Latina. Paris.

Cronbach, L. J. (1951). Coefficient alpha and the internal structure of tests. Psychometrika, 16(3), $297-334$. doi: https://doi.org/10.1007/BF02310555

Delgado Vásquez, A. (2004). Tesis: Relación entre los estilos de aprendizaje y los estilos de pensamiento en estudiantes de maestría considerando las especialidades profesionales y el tipo de universidad.

Ding, C. y Hershberger, S. (2002). Assessing content validity and content equivalence using structural equation modeling. Structural Equation Modeling: A Multidisciplinary Journal, 9 (2), 283-297.

Escurra, M. (1991). Adaptación del Inventario de estilos de Aprendizaje de Kolb en estudiantes de psicología pertenecientes a diferentes universidades de Lima Metropolitana. Tesis para optar el título profesional de psicólogo. UNMSM, Lima.

Fundación Telefónica (2105) Las MOOC en la educación del futuro: la digitalización de la formación. Barcelona: Editorial Ariel

Gallego J., Gámiz V. y Gutiérrez E. (2015) Tendencias en la evaluación del aprendizaje en cursos en línea masivos y abiertos, Educación XX1, 18 (2),77-96. doi: http://dx.doi.org/10.5944/educxx1.12935

García E., Barrantes C., Ureña A. y Mora F. (2015). Relación entre estilo de aprendizaje y rendimiento académico en estudiantes de farmacia de la Universidad de Costa Rica. RIDU, 9(2), 5.

González, M.R. (1983). Influencia de la naturaleza de los estudios universitarios en los estilos de aprendizaje de los sujetos. Tesis Doctoral, Universidad Complutense de Madrid. Facultad de Psicología.

González S., Del Pozo F., Paredes W. y Del Pozo H. (2018). Los MOOC: tecnología y pedagogía emergente para la democratización del conocimiento. REV. PERSPECTIVA 19 (2), 215-224.

González M., Patarroyo N. y Carreño C. (2017). El principio de justicia en el aula y la responsabilidad moral del docente, frente a los estilos de aprendizaje Revista de investigación, desarrollo e innovación, 7(2), 241-253. doi: https://doi.org/10.19053/20278306.v7.n2.2017.4497

Gordillo I., Antelo I., García F. y Sayago A. (2013). Estilos de aprendizaje del alumnado de Psicopedagogía y su implicación en el uso de las TIC y aprendizaje colaborativo. Revista de Educación a Distancia, (35).

Gregori P., Martínez V. y Moyano J. (2018). Basic actions to reduce dropout rates in distance learning. Evaluationand programplanning,66,48-52.doi:https://doi.org/10.1016/j.evalprogplan.2017.10.004

Gutarra Palomino C. (2015). Tesis Estilos de aprendizaje en las alumnas del 5 to grado de educación secundaria de la I. E Nuestra Señora de Cocharcas-Huancayo.

Hernández, R., Fernández, C. y Baptista, P. (2010). Metodología de la Investigación. $2^{a}$ Edición. México: 
Mc Graw Hill..

Herrera L. y De la Hoz A. (2014). El ciclo de aprendizaje experiencial en la enseñanza. Caso ESUMER-GIZ: Seminario sobre cooperación y relaciones comerciales con Alemania. Nodos y Nudos, 4(37). doi: https://doi.org/10.17227/01224328.3128

Heuser A., Rioul O. y Guilley S. (2014) A Theoretical Study of Kolmogorov-Smirnov Distinguishers. In: Prouff E. (eds) Constructive Side-Channel Analysis and Secure Design. Lecture Notes in Computer Science, vol 8622(1). doi: https://doi.org/10.1007/978-3-319-10175-0_2

Kolb D. (1983) Experiential Learning: Experience as the Source of Learning and Development. USA: Prentice Hall.

Kolb D., Boyatzis R. y Mainemelis C. (1999). Experiential learning Theory: Previous Research and New Directions. Perspectives on thinking, learning, and cognitive styles, 1(8), 227-247.

Lawshe, C. H. (1975). A quantitative approach to content validity. Personnel Psychology, 28(4), 563-575. doi: http://dx.doi.org/10.1111/j.1744-6570.1975.tb01393.x

Martín, A. (2003). Estilos de aprendizaje en la vejez. Un estudio a la luz de la teoría del aprendizaje experiencial. Revista Española de Geriatría y Gerontología, 38(5), 258-265. doi: https://doi. org/10.1016/S0211-139X(03)74896-3

Maurandi-López A., Del Río Alonso L., González-Vidal A., Ferre M. y Hernández A. (2019). Fundamentos Estadísticos para Investigación. Introducción a R y Modelos (Versión 2.0). doi: http://doi. org/10.5281/zenodo.2628915

Poy, R. y Gonzales-Aguilar A. (2014). Factores de éxito de los MOOC: algunas consideraciones críticas. RISTI-Revista Ibérica de Sistemas e Tecnologias de Informação, (SPE1), 105-118.

Rodríguez Cepeda R. (2018) Los modelos de aprendizaje de Kolb, Honey y Mumford: implicaciones para la educación en ciencias. Sophia, 14(1), 51-64. doi: http://dx.doi.org/10.18634/sophiaj.14v.1i.698.

Ruiz, C. (2015) "El MOOC: ¿un modelo alternativo para la educación universitaria?", Apertura. Revista de innovación educativa, 7(2), 1-14. Universidad de Guadalajara.

Ruiz, Guillermo (2013). La teoría de la experiencia de John Dewey: significación histórica y vigencia en el debate teórico contemporáneo. Foro de Educación, 11(15). doi: http://dx.doi.org/10.14516/ fde.2013.011.015.005

Sánchez C. y Reyes C. (2015). Metodología y Diseños en la investigación científica. Lima - Perú: Editorial Visión Universitaria.

Sánchez M. y Prendes M. (2014). La participación del alumnado en los cursos masivos (MOOC). En II Congreso Internacional de Innovación Docente.

Sandoval C., Morales M., Hernández R. y Amado-Salvatierra H. (2018) Estrategias para la reducción de la deserción en los MOOC: Experiencia del MOOC Marketing Digital.

Torres J., Infante A., Valdiviezo P. (2014) Los MOOC y la masificación personalizada. Profesorado. Revista de Currículum y Formación de Profesorado, 18(1),63-72. 